\title{
Multivariate analysis of early surgical management factors affecting posttraumatic penoscrotal avulsion injury: a level I trauma center study
}

Min Ji Kim, Dong Hwan Lee, Dong Ha Park and II Jae Lee * (1)

\begin{abstract}
Background: To conduct an accurate evaluation of patients presenting with posttraumatic penoscrotal injuries, and to formulate a treatment algorithm based on this assessment.

Methods: We conducted a retrospective chart review study. Patients with penoscrotal defects admitted to our level I trauma center from 2017 to 2019 were evaluated. The Braden scale score was used for wound evaluation and the Korean patient classification system (KPCS) was used for assessment of severity. Univariate and multivariate analyses were performed for potential risk factors associated with early surgical management.
\end{abstract}

Results: In total, there were 58 male patients, and the average Braden scale score was $12.08 \pm 2.54$, with the scrotum (36.20\%), and the penile shaft (32.76\%) being popular sites for injuries. The wounds requiring surgical treatment were $20.68 \%(n=12)$, with local flaps (33.33\%) being most commonly used. The significant predictors of advanced wounds which required surgical treatment were old age $(p=0.026$, odds ratio [OR] 8.238), orthopedic combined injuries ( $p=0.044, \mathrm{OR} 1.088)$, intubation $(p=0.018, \mathrm{OR} 9.625)$, restraint $(p=0.036, \mathrm{OR} 0.157)$ and blood transfusion $(p<0.001$, OR 2.462).

Conclusion: In multiple trauma patients, penoscrotal defects caused by high-speed trauma are an important matter of concern. Specifically, patients with combined skeletal injuries or requiring respiratory care were prone to advanced wounds. We proposed a five-category algorithm to manage such patients, which included severity of the patient's condition, respiration, hemodynamic status, comorbidity, and immobilization. Additionally, inter-departmental cooperation and active intervention by plastic surgeons is needed for the comprehensive treatment of such injuries.

Trial registration This study was performed in line with the principles of the Declaration of Helsinki. The study and all its protocols were approved by the institutional review board of Ajou Medical Center (approval no. AJIRB-MEDMDB-17-254). The need for informed consent was waived by the institutional review board of our hospital due to the retrospective design of the study.

Keywords: Penoscrotal avulsion, Trauma, Wound management, Quality improvement, Wound and injury

*Correspondence: i00325@live.co.kr

Department of Plastic and Reconstructive Surgery, Ajou University School of Medicine, 164, World cup-ro, Yeongtong-gu, Suwon 16499, Republic of Korea

\section{Background}

In multiple severe trauma patients, high-speed mechanism injuries in accidents can cause penoscrotal defects. The importance of this region for the body's urologic and sexual functions is well known, and the preservation of 
the structure and function of the penoscrotal region is integral to the patient's quality of life [1]. In some disease involving penoscrotal defects such as Fournier's gangrene, the physician has to focus on wound management, often necessitating early referral to a reconstructive specialist [2]. However, severely trauma patients admitted to a level I trauma center differ from patients suffering from a single disease. Recently, cancer-related mortality has decreased by $20 \%$ (1991-2009); however, trauma-related mortality has increased by $24 \%(1990-2010)[3,4]$. These patients are managed according to a structured resuscitation protocol. Furthermore, these multiple trauma patients may present with an edematous perineum, hiding a penoscrotal avulsion injury. In such critical conditions, such wounds that are not immediately obvious, can easily be overlooked even by plastic surgeons.

For the management of penoscrotal avulsion injuries, a comprehensive evaluation of the patient's status is needed. Surgical reconstruction of a penoscrotal lesion is not simple. The goal is to provide wound healing, adequate function, and an acceptable appearance [5]. To date, recent studies have underestimated such injuries; thus, information regarding these injuries is scarce. The purpose of this study was to provide an accurate evaluation of trauma patients with penoscrotal injuries and to propose a treatment algorithm based on this assessment.

\section{Methods}

We retrospectively recruited patients who were admitted to our level I trauma center, with trauma-related penoscrotal defects between 2017 and 2019. The following demographic information was collected; age, sex, cause of injury, combined injuries, management protocols including respiratory care, intubation, nutrition, restraint, range of motion, and whether or not they were alive at presentation. The wound characteristics were evaluated according to severity, anatomical lesion, defect size, management technique, operative details, and complications. The Braden scale score was used for wound evaluation, and the KPCS (Korean patient classification system) was used for the assessment for patient severity [6-9]. The Braden Scale assesses risk using six different risk factors: sensory perception, the ability to respond meaningfully to pressure-related discomfort; moisture, defined as the degree to which skin is exposed to moisture; activity, degree of physical activity; mobility, defined as the ability to change and control body position; nutrition, usual food intake pattern; and friction and shear [6, $10,11]$. The Braden scale is widely used to evaluate the risk of sores. It has several components to enable various aspects of wound evaluation. However, it can be used to evaluate other wounds apart from sores. KPCS consisted of 12 areas, 50 nursing activities, and 73 items (factors for general nursing care) [12]. It was developed based on the Workload management system for nurses (WMSN) of the USA. There are many reports of its validity and reliability; therefore, this classification has been included in the basic tools used in tertiary hospitals in Korea [13-15]. Wound dimension was assessed using Image) (National Institutes of Health, USA) [16]. For each photographic image, measurements were calibrated using pixels at the margin of the wound comparing with the entire genital area. The wound size was calculated using the percentage of the total genital area according to pixel count. Statistical analysis was performed using Statistical Package for the Social Sciences (SPSS) for Windows version 18.0 (SPSS Inc., Chicago, IL, USA). Data are presented as means \pm 2 standard deviations. Statistical significance was accepted at $p<0.05$. Factors that predicted surgically demanding and advanced wounds were identified through a univariate analysis. A surgically demanding wound is defined as an open wound that cannot be expected to heal completely with conservative dressing treatment. Such wounds are expected to require surgical intervention to enable healing. Advanced wounds are a sub-category of surgically demanding wounds. These wounds require surgical treatment, but the focus of treatment is tissue reconstruction. Therefore, simple wounds that require only debridement are excluded from this category. Variables with a $p$-value $<0.05$ were included in the multivariate analysis. Backward-stepwise logistic regression analysis was performed to identify risk factors for trauma-related advanced penoscrotal defects. The need for informed consent was waived by the Institutional Review Board because of the retrospective nature of the study. However, photographic authorization and release consent were obtained from all participants, including parental consent for adolescent patients. This study strictly adhered to Declaration of Helsinki principles.

\section{Results}

A total of 58 patients were included in this retrospective study. All the patients were male and had an average age of 59.72 years (range, 40-78). Mean follow-up period was 13.74months (range, 5.5-22).Intensive care unit patient severity, as assessed by KPCS, had mean values of $4.41 \pm 1.22$. Wound severity assessed by Braden scale had an average of 12.08, and high-risk patients for whom scale was lower than 12 were $65.52 \%$. The most common cause of injury was a fall, followed by traffic accidents, each contributing to 37.93 and $22.41 \%$ of the injuries, respectively. Wound extension was classified on the basis of anatomical sites, and scrotum and shaft were the most commonly injured sires, at 36.20 and $32.76 \%$ respectively [17]. On comparing with the total genital area, the 
involved penoscrotal defect portion was $55.80 \pm 30.45 \%$ (Table 1).

Comorbidities were classified into nine areas: brain, orthopedic, spine, facial, vascular, lung, intraorgan, and soft tissue comorbidities and acute respiratory distress syndrome. The most common combined injury was orthopedic injury (65.62\%), followed by intraorgan injury (37.93\%), and spine injury (34.48\%). Extensive soft tissue injuries were found in $20.69 \%$, but these injuries did not involve genital wounds. Among 58 patients, $75.85 \%$ survived, however, 15 patients died in spite of active resuscitation at a professional trauma center. The rate of ventilator assistance was 44.83 , and $91.38 \%$ of the patients received a blood transfusion during the hospital stay. Some hemodynamic indicators were evaluated; the collected mean hemoglobin level was $11.41 \pm 1.80$ and the mean creatinine level was $1.22 \pm 0.83$ (Table 2).

Among the 58 patients, 12 received surgical wound management. The remaining 47 patients were treated with conservative management, which included aseptic dressing, negative pressure vacuum dressing, and growth factor ingredient medication-assisted dressing.

Table 1 Patient demographics

\begin{tabular}{|c|c|}
\hline Variable & Values \\
\hline No. of subjects & 58 \\
\hline Age (years) ${ }^{\mathrm{a}}$ & $59.72 \pm 19.25$ \\
\hline \multicolumn{2}{|l|}{$\operatorname{Sex}(n)$} \\
\hline M & 58 \\
\hline Severity ${ }^{b}$ & $4.41 \pm 1.22$ \\
\hline Braden scale ${ }^{a}$ & $12.08 \pm 2.54$ \\
\hline \multicolumn{2}{|l|}{ Braden scale $(n, \%)$} \\
\hline$\leq 18$ & $38(65.521)$ \\
\hline \multicolumn{2}{|l|}{ Cause of injury $(n, \%)$} \\
\hline Fall & $22(37.93)$ \\
\hline Traffic accident & $13(22.41)$ \\
\hline Motorcycle accident & $9(15.51)$ \\
\hline Rolling injury & $4(6.90)$ \\
\hline Crushing injury & $5(8.62)$ \\
\hline Others & $5(8.62)$ \\
\hline \multicolumn{2}{|l|}{ Site of lesion (n, \%) } \\
\hline Glans & $2(3.44)$ \\
\hline Corona & $1(1.72)$ \\
\hline Foreskin & $8(1.38)$ \\
\hline Shaft & $19(32.76)$ \\
\hline Root & $7(12.07)$ \\
\hline Scrotum & $21(36.20)$ \\
\hline Size of defect (\% of total genital area) & $55.80 \pm 30.45$ \\
\hline Follow up months ${ }^{a}$ & $13.74 \pm 8.20$ \\
\hline
\end{tabular}

a Mean $\pm 2 S D$

b Korean Patient Classification System for Nurses
Table 2 General comorbidities and hemodynamic status in the study population

\begin{tabular}{ll}
\hline Variable & Values \\
\hline Combined injury (n, \%) & \\
Brain injury & $15(25.86)$ \\
Orthopedic injury & $38(65.62)$ \\
Spine injury & $20(34.48)$ \\
Facial bone injury & $7(12.07)$ \\
Vascular injury & $15(25.86)$ \\
Lung injury & $19(15.51)$ \\
Intraorgan injury & $22(37.93)$ \\
Extensive soft tissue injury (except genital) & $12(20.69)$ \\
Acute respiratory distress syndrome & $10(17.24)$ \\
Intubation ( $n$, \%) & $26(44.83)$ \\
Absolute bed rest (n, \%) & $8(13.79)$ \\
Restrain ( $n$, \%) & $24(41.37)$ \\
Total parenteral nutrition (n, \%) & $25(43.10)$ \\
Transfusion ( $n$, \%) & $53(91.38)$ \\
Initial hemodynamic status ${ }^{\text {a }}$ & \\
Hb & $11.41 \pm 1.80$ \\
Albumin & $3.32 \pm 0.69$ \\
Bun & $17.42 \pm 8.87$ \\
Creatinine & $1.22 \pm 0.83$ \\
Survival (n, \%) & $44(75.86)$ \\
Expire (n, \%) & $15(25.86)$ \\
\hline a Mean $\pm 2 S D$ &
\end{tabular}

a Mean $\pm 2 S D$

The operative options ranged from debridements and local flaps to skin grafts and free flaps (Figs. 1, 2). The most common modality used was local flap in 33.33\% of patients, followed by debridement in $25 \%$, and free flap in $25 \%$. Severe complications were not noted, and only minor complications, including partial necrosis, were noted (Table 3).

Univariate analysis identified several clinical variables associated with the development of a surgically demanding advanced wound. Age, Braden scale, orthopedic injury, intraorgan injury, extensive soft tissue injury, intubation, absolute bed rest, restraint, transfusion, hemoglobin, blood urea nitrogen, and survival status were found to be associated with advanced wounds. Multivariate logistic regression analysis of these variables showed that old age ( $p=0.026$, OR 8.238), orthopedic combined injury ( $p=0.044$, OR 1.088), intubation $(p=0.018$, OR 9.625), restraint $(p=0.036$, OR 0.157$)$, and transfusion $(p<0.001$, OR 2.462), were significant predictors of advanced wounds which would require surgical treatment (Table 4). 

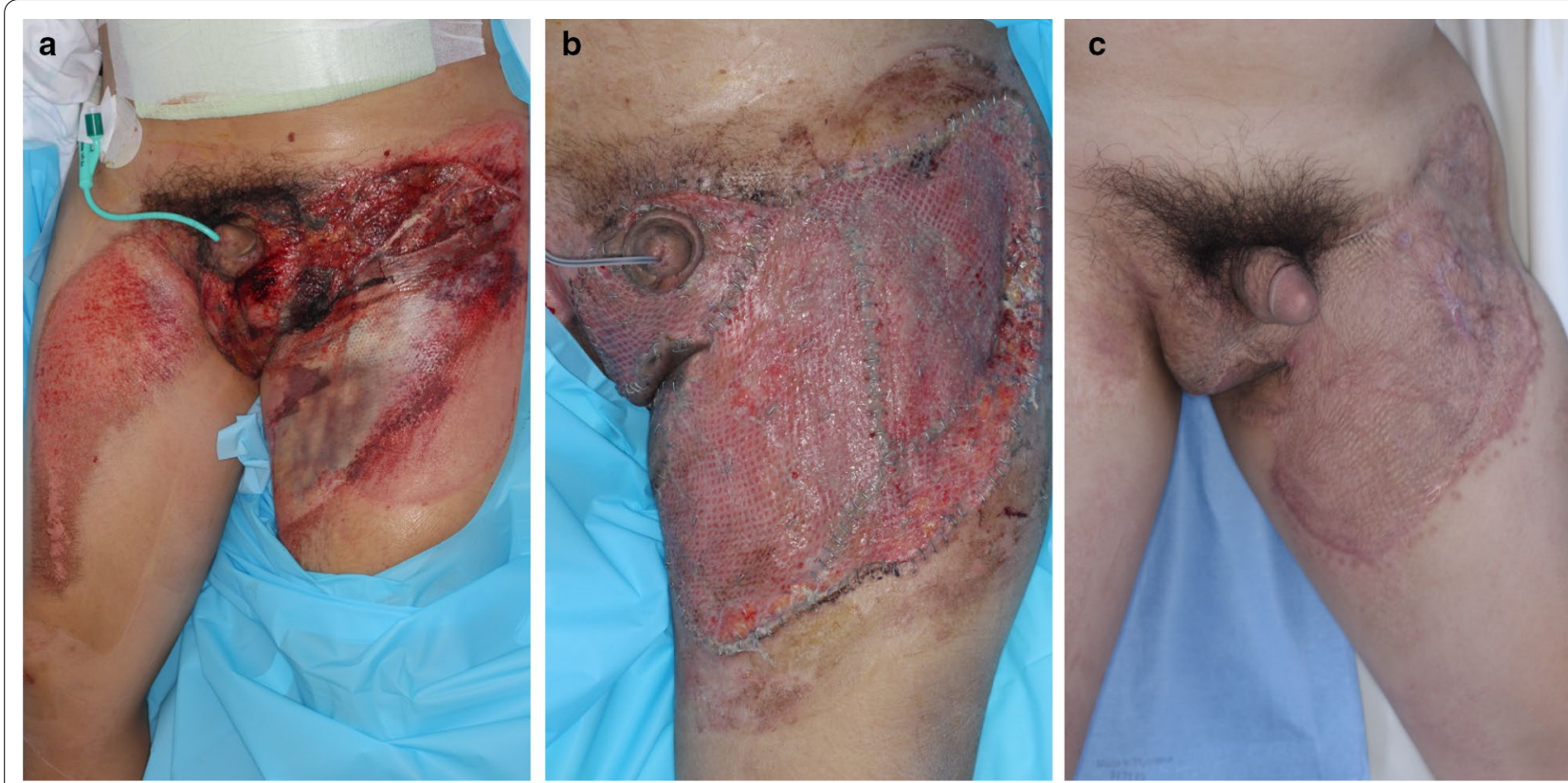

Fig. 1 Representative case of a penoscrotal injury where successful surgical reconstruction was achieved. a The 36-year-old male pedestrian with no underlying conditions was hit by a vehicle and admitted for soft tissue injury involving the scrotum and thigh, an ankle fracture, and a pelvic bone fracture. He was transfused for massive blood loss, but not intubated. The urologist confirmed that the wound did not invade the testicles and he had no problems with sexual function and urination. b After several debridements, he underwent a split-thickness skin graft. c One year later, he had discomfort due to scar contracture in his inguinal area, and the scar contracture was released with a Z-plasty. According to the five-category algorithm, he had orthopedic injury, required restraint, and transfusion — a total of three factors that could signify an advanced wound
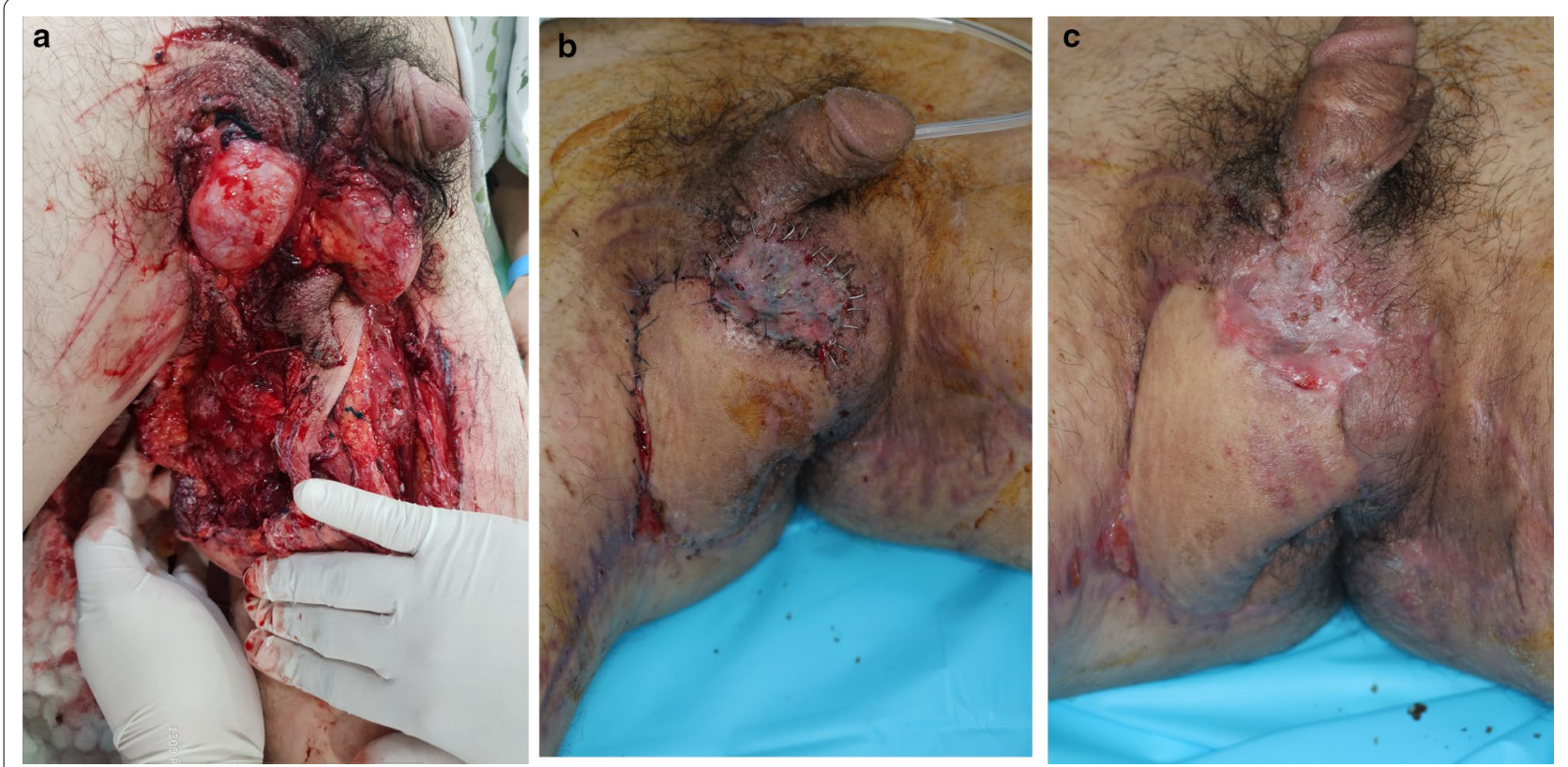

Fig. 2 Representative case of penoscrotal injury where successful surgical reconstruction was achieved. a A 34-year-old man with no underlying disease admitted for soft tissue injuries including the scrotum, bilateral thighs, and perineum, because his thigh rolled into a rolling machine. His vital signs were stable. There was no testicular and anal injury. $\mathbf{b}$ After several debridements, a medial circumflex artery perforator-based local flap rotation, and split-thickness skin grafting were performed. c After 1 month, the wound was well healed. According to the five-category algorithm, he had intubation, required restraint, and transfusion - a total of three factors that could signify an advanced wound 
Table 3 Outcome of surgical, non-surgical management of penoscrotal defect

\begin{tabular}{ll}
\hline Variable & Values \\
\hline Conservative management $(n, \%)$ & $47(81.03)$ \\
Surgical management $(\mathrm{n}, \%)$ & $12(20.68)$ \\
Operative option & \\
Debridement & $3(25.00)$ \\
Local flap & $4(33.33)$ \\
Skin graft & $2(16.67)$ \\
Free flap & $3(25.00)$ \\
Surgical complication & \\
Infection & - \\
Partial necrosis & $3(25.00)$ \\
Total necrosis & - \\
\hline
\end{tabular}

\section{Discussion}

The development of high-speed machines and an advanced building industry has changed the presentation of trauma patients and it leads to associated industrial accidents as well as traffic accidents. Such trauma can involve every part of the body, including the penoscrotal area. Owing to their concealed anatomical locations, such injuries can go easily undetected, further delaying treatment. There are few comments on the mechanism of development of penoscrotal injuries in a trauma patient, besides a direct injury of the perineal area from the trauma. In rotation machines, the capturing force of the machine creates torsion and leads to traction of the area $[18,19]$. In falls, direct trauma to the falling side causes friction burns which lead to soft tissue injuries. If the scrotal skin loss is less than $50 \%$, it can often be closed immediately after the trauma [19]. The depth of injury can involve damage to the cavernous bodies, spongy body, or testes [20]. Following blood loss, infection and lymphedema can lead to the development of an irreversible wound.

In this study, our final proposal was an assessment algorithm for trauma-related penoscrotal avulsion injury (Fig. 3). We composed a five-category algorithm, based on our multivariate clinical risk evaluation. The categories were patient severity, respiration, hemodynamic status, comorbidities, and immobilization. In this algorithm, the main factors that contribute to advanced penoscrotal injury are composed of patient stability, immobilization,

Table 4 Variables associated with surgical management of trauma-induced penoscrotal defect

\begin{tabular}{|c|c|c|c|c|c|}
\hline \multirow[t]{2}{*}{ Variables } & \multicolumn{2}{|c|}{ Univariate analysis } & \multirow[b]{2}{*}{ Odds ratio } & \multicolumn{2}{|c|}{ Multivariate analysis } \\
\hline & Odds ratio & $p$-value & & $95 \% \mathrm{Cl}$ & $p$-value \\
\hline Age & 1.019 & $0.006^{\mathrm{a}}$ & 8.238 & $0.000,10.944$ & $0.026^{\mathrm{a}}$ \\
\hline Severity & 0.727 & 0.210 & - & - & - \\
\hline Braden scale & 0.548 & $0.047^{\mathrm{a}}$ & 2.462 & $1.497,4.049$ & 0.877 \\
\hline Cause of injury & 1.205 & 0.057 & - & - & - \\
\hline Brain injury & 0.339 & 0.142 & - & - & - \\
\hline Orthopedic injury & 0.460 & $0.048^{\mathrm{a}}$ & 1.088 & $0.008,1.932$ & $0.044^{\mathrm{a}}$ \\
\hline Spine injury & 0.374 & 0.145 & - & - & - \\
\hline Facial injury & 0.693 & 0.683 & - & - & - \\
\hline Vascular injury & 1.019 & 0.978 & - & - & - \\
\hline Lung injury & 0.577 & 0.394 & - & - & - \\
\hline Intraorgan injury & 1.231 & $0.018^{\mathrm{a}}$ & 3.087 & $0.010,9.724$ & 0.402 \\
\hline Extensive soft tissue injury & 1.933 & $<0.001^{\mathrm{a}}$ & 6.772 & $1.258,13.578$ & 0.210 \\
\hline ARDS & 2.617 & 0.284 & - & - & - \\
\hline Intubation & 2.903 & $0.021^{\mathrm{a}}$ & 9.625 & $1.466,63.549$ & $0.018^{\mathrm{a}}$ \\
\hline Absolute bed rest & 6.429 & $0.028^{\mathrm{a}}$ & 1.657 & $0.147,3.488$ & 0.344 \\
\hline Restrain & 0.253 & $0.042^{\mathrm{a}}$ & 1.157 & $0.028,0.888$ & $0.036^{\mathrm{a}}$ \\
\hline Total parenteral nutrition & 5.040 & 0.155 & - & - & - \\
\hline Transfusion & 2.201 & $0.001^{\mathrm{a}}$ & 2.462 & $1.497,4.049$ & $<0.001^{\mathrm{a}}$ \\
\hline $\mathrm{Hb}$ & 0.229 & $0.001^{\mathrm{a}}$ & 1.050 & $0.733,1.502$ & 0.791 \\
\hline Albumin & 1.821 & 0.285 & - & - & - \\
\hline BUN & 1.254 & $0.020^{\mathrm{a}}$ & 0.818 & $0.691,0.969$ & 0.351 \\
\hline Creatinine & 0.002 & 0.060 & - & - & - \\
\hline Expire & 0.039 & $0.022^{\mathrm{a}}$ & 2.007 & $0.261,3.988$ & 0.157 \\
\hline
\end{tabular}

a Statistically significant; $A R D S$ acute respiratory distress syndrome, $B U N$ blood urea nitrogen 


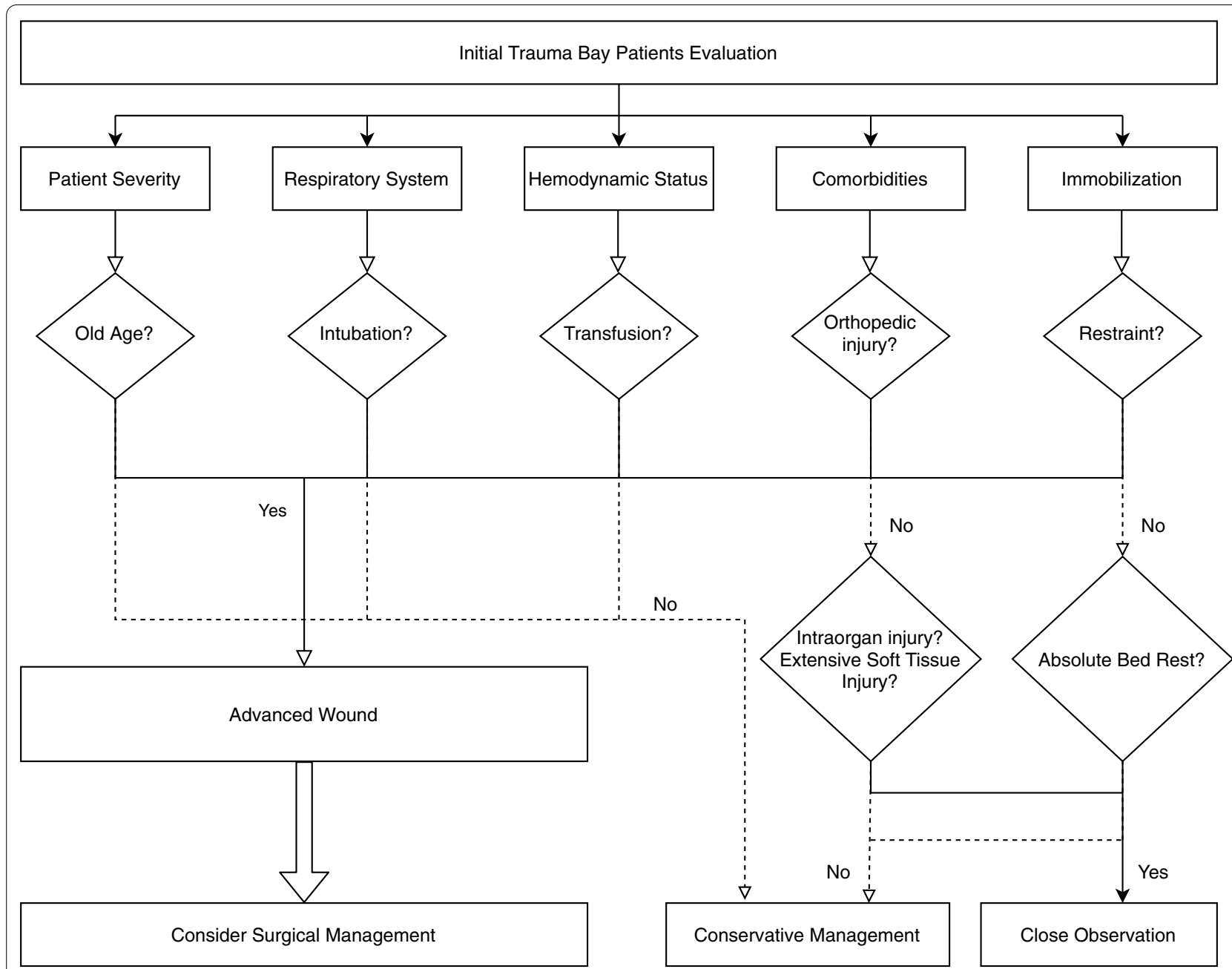

Fig. 3 Treatment algorithm for posttraumatic penoscrotal injury

and oxygen insufficiency. Orthopedic injury and restraint contribute to immobilization, and this can lead to delayed wound healing and wound deepening. Insufficient respiration and blood loss contribute to oxygen insufficiency, which leads to wound necrosis and aggravation of the edema. Through this algorithm, we want to emphasize that such patients should not simply be referred to the plastic surgeon. Initially, the critical care team should get involved with the wound evaluation and discuss management with other teams. The categorized component starts from the initial patient evaluation. Initially in the emergency room, a comprehensive wound approach is required for successful reconstruction. During the patient's hospital stay, there might be further possibilities for the critical care team to get involved. However, our study stated that when the algorithm is first applied, we simply need to check the wound status to determine whether the wound is likely to progress into an advanced wound.
The details of the treatment algorithm are described below. Firstly, in multiple trauma patients, the initial hemodynamic status should be assessed. Various laboratory findings and hemodynamic indicators help us decide whether resuscitation treatments should be started immediately or later. Hemodynamic instability should be considered first; if the patient is persistently unstable, stabilization without active wound management should be considered. Massive blood loss usually requires transfusion; thus, integration of transfusion management should be considered. The next step is the severity of the patient's condition, which should be considered with respect to their age. Respiratory care with intubation, in our experience, increased the risk of penoscrotal injury with a highly advanced wound status; thus, active surgical management should be considered. In multiple trauma patients, their additive injury status leads to further problems. Thus, a categorized evaluation is needed. In particular, combined 
orthopedic injuries were associated with high rates of advanced penoscrotal wounds which required special attention. Moreover, intraorgan injuries and extensive soft tissue injuries are often associated with advanced penoscrotal wounds; however, no statistical significance was noted in our study. Finally, based on these five categories of evaluation in trauma patients, we aim to detect penoscrotal wounds earlier and start active interventions.

Why is the early diagnosis of surgical prone penoscrotal defects in trauma patients important for reconstruction? Extensive penoscrotal defects ultimately require surgical reconstruction. However, anatomically the lesion penoscrotal area, there is a presence of circumferential muscle and its flexible skin texture has to be dealt with in a very particular attention. Especially erectile function should be reproduced via functional reconstruction. These days, sensate flap reconstruction is possible, hence one should not delay a referral. In our study, the most common surgical option was a local flap. The range of local flaps includes skin advancement using inguinal to upper thigh skin, local fasciocutaneous flaps, and musculocutaneous flaps. The next step for a successful reconstruction is choosing a reconstructive option. This decision is best made after the serial debridement. Once we follow the penoscrotal defect from the initial status without infection, the debrided necrotic tissue can help the surgeon make the decision. Once healthy granulation tissue is observed, we can apply the skin graft, which works best on the scrotum and shaft. As these areas have very thin epithelium this vacuum-assisted treatment is very helpful. However, complex anatomical lesions and wounds extended to the inguinal area are limited with respect to formation of granulation tissue. In this case, we used local flap advancement by using the surrounding abdominal or thigh soft tissue. However, in cases of exposed tendon or vessel, a free flap is required. Functionally and aesthetically pleasing reconstructions are now possible. However, while choosing an appropriate method of surgical reconstruction, we need to take the patient's overall status into consideration.

The limitations of our research are as follows. Owing to the nature of a level I trauma center, the severity of the patients' condition is relatively high, which could have contributed to the higher mortality seen in our study compared to those in other studies. The resuscitation rates such as respiratory resuscitation and transfusion rates were also very high. However, the mortality itself was not directly associated with the severity of the penoscrotal injury. Therefore, there was a limitation in the association between the nature of the trauma center itself with the severity of the wounds. Through this study, we hope that the discovery algorithm used to detect penoscrotal injuries in traumatic patients, will now include more than just an assessment of wound complexity.

\section{Conclusions}

In multiple trauma patients, due to the requirement of comprehensive management, penoscrotal defects caused by trauma are prone to going undetected. However, the functional and psychological importance of this area demands definitive attention and care from the physician, especially for patients with combined skeletal injuries or those on respiratory care who are likely to have advanced wounds warranting surgical treatment. We proposed a five-category algorithm to manage penoscrotal injuries in trauma patients. Inter-departmental cooperation and active intervention by plastic surgeons is needed for comprehensive treatment. We hope the proposed algorithm will help in the early detection of trauma-related penoscrotal injuries and promote early referral for their active surgical or non-surgical management.

\section{Abbreviations \\ KPCS: Korean patient classification system. \\ Acknowledgements \\ The authors would like to thank everyone who participated in the manage- ment and for taking care of the trauma patients in Ajou trauma center.}

\section{Authors' contributions}

MJK wrote the original draft. DHL and DHP performed data curation. IJL was involved in visualization. MJK and IJL was responsible for project administration. All authors read and approved the final manuscript.

\section{Funding}

No funding was received for this study.

\section{Availability of data and materials}

The datasets used and/or analysed during the current study available from the corresponding author on reasonable request.

\section{Ethics approval and consent to participate}

This study was performed in line with the principles of the Declaration of Helsinki. The study and all its protocols were approved by the institutional review board of Ajou Medical Center (approval no. AJIRB-MED-MDB-17-254).

The need for informed consent was waived by the institutional review board of our hospital due to the retrospective design of the study.

\section{Consent for publication}

We received the photo release consent from the all included patients and they agreed for the publication of identifying images in academic purpose.

\section{Competing interests}

The authors declare that they have no competing interests.

Received: 23 August 2020 Accepted: 25 November 2020 Published online: 07 January 2021 


\section{References}

1. Tran NV. Scrotal and perineal reconstruction. In: Seminars in plastic surgery. Thieme Medical Publishers, 2011. p. 213.

2. Chen S-Y, et al. Fournier gangrene: a review of 41 patients and strategies for reconstruction. Ann Plast Surg. 2010;64(6):765-9.

3. Benjamin EJ, et al. Heart disease and stroke statistics - 2018 update: a report from the American Heart Association. Circulation. 2018.

4. Jung KW, et al. Cancer statistics in Korea: incidence, mortality, survival, and prevalence in 2016. Cancer Res Treat. 2019:51(2):417.

5. Lee SH, Rah DK, Lee WJ. Penoscrotal reconstruction with gracilis muscle flap and internal pudendal artery perforator flap transposition. Urology. 2012;79(6):1390-6.

6. Iranmanesh S, Rafiei H, Sabzevari S. Relationship between Braden scale score and pressure ulcer development in patients admitted in trauma intensive care unit. Int Wound J. 2012;9(3):248-52.

7. Brindle CT, et al. Outliers to the Braden scale: identifying high-risk ICU patients and the results of prophylactic dressing use. World Counc Enteros Ther J. 2010;30(1):11.

8. Song YJ. The south Korean health care system. Japan Med Assoc J. 2009:52(3):206-9.

9. Lee $\mathrm{H}$, et al. Application of different prognostic scoring systems and comparison of the FAB and WHO classifications in Korean patients with myelodysplastic syndrome. Leukemia. 2003;17(2):305-13.

10. Baldwin KM, Ziegler SM. Pressure ulcer risk following critical traumatic injury. Adv Wound Care. 1998;11(4):168-75.

11. Griswold LH, Griffin RL, Swain T, Kerby JD. Validity of the Braden scale in grading pressure ulcers in trauma and burn patients. J Surg Res. 2017;219:151-7.
12. Bong M, Kim K, Kim L, Jeong Y, An Y. Availability of patient classification using clinical data. In: NI 2012: 11th international congress on nursing informatics, June 23-27, 2012. Montreal: American Medical Informatics Association; 2012

13. Seago JA. A comparison of two patient classification instruments in an acute care hospital. J Nurs Adm. 2002;32(5):243-9.

14. Molter NC, Molter NC. Workload management system for nurses: application to the burn unit. J Burn Care Rehabil. 1990;11(3):267-74.

15. Yoo C-S, Kwon E-O, Kim S-H, Cho Y. Reliability, validity, and conversion index of the workload management system for critical care nurses (WMSCN). J Korean. Crit Care Nurs. 2009;2:48-57.

16. Chang AC, Dearman B, Greenwood JE. A comparison of wound area measurement techniques: visitrak versus photography. Eplasty. 2011;11.

17. Keays MA, Dave S. Current hypospadias management: diagnosis, surgical management, and long-term patient-centred outcomes. Can Urol Assoc J. 2017;11(1-2 Suppl1):S48.

18. Tripathi FM, et al. Traumatic avulsion of penile and scrotal skin. Br J Plast Surg. 1982;35(3):302-3.

19. Masters FW, Robinson DW. The treatment of avulsions of the male genitalia. J Trauma Acute Care Surg. 1968;8:430-8.

20. Finical SJ, Arnold PG. Care of the degloved penis and scrotum: a 25-year experience. Plast Reconstr Surg. 1999;104(7):2074-8.

\section{Publisher's Note}

Springer Nature remains neutral with regard to jurisdictional claims in published maps and institutional affiliations.
Ready to submit your research? Choose BMC and benefit from:

- fast, convenient online submission

- thorough peer review by experienced researchers in your field

- rapid publication on acceptance

- support for research data, including large and complex data types

- gold Open Access which fosters wider collaboration and increased citations

- maximum visibility for your research: over $100 \mathrm{M}$ website views per year

At $\mathrm{BMC}$, research is always in progress.

Learn more biomedcentral.com/submissions 